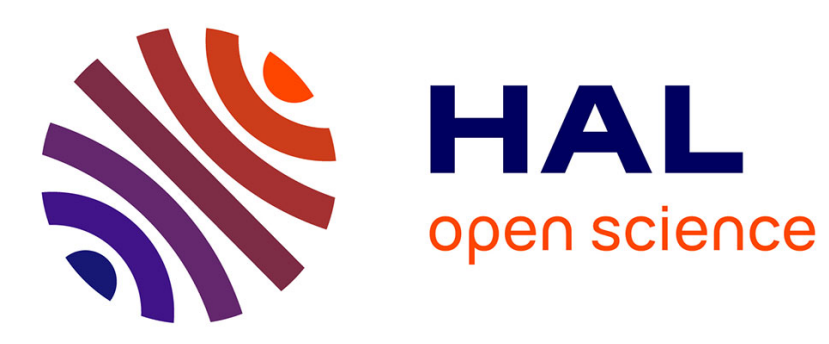

\title{
Characterization of massive magnetic parts with a dedicated device
}

Marc Borsenberger, Abdelkader Benabou, Cyrille Baudouin, Régis Bigot, Pierre Faverolle, Jean-Claude Mipo

\section{- To cite this version:}

Marc Borsenberger, Abdelkader Benabou, Cyrille Baudouin, Régis Bigot, Pierre Faverolle, et al.. Characterization of massive magnetic parts with a dedicated device. International Journal of Applied Electromagnetics and Mechanics, 2018, 57 (4), pp.457-472. 10.3233/jae-170108 . hal-02288807

\section{HAL Id: hal-02288807 https://hal.science/hal-02288807}

Submitted on 16 Sep 2019

HAL is a multi-disciplinary open access archive for the deposit and dissemination of scientific research documents, whether they are published or not. The documents may come from teaching and research institutions in France or abroad, or from public or private research centers.
L'archive ouverte pluridisciplinaire HAL, est destinée au dépôt et à la diffusion de documents scientifiques de niveau recherche, publiés ou non, émanant des établissements d'enseignement et de recherche français ou étrangers, des laboratoires publics ou privés. 


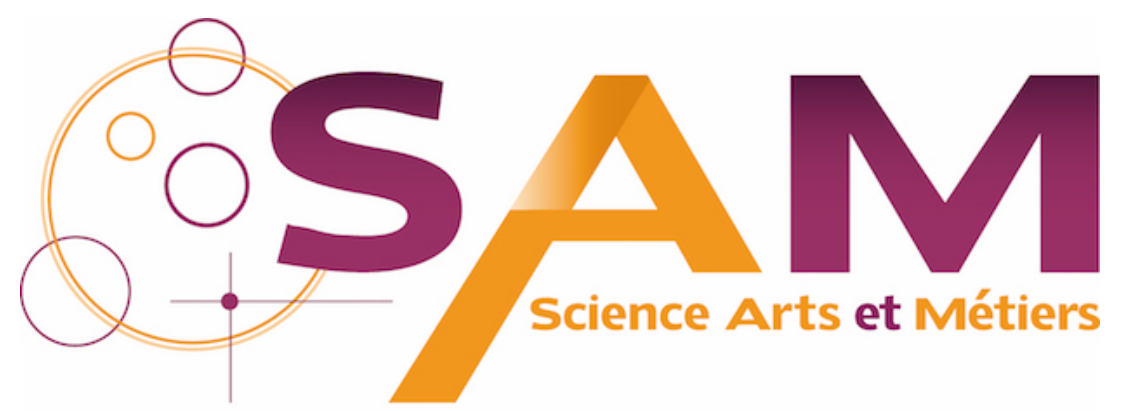

Archive Ouverte - Open Repository

\section{Science Arts \& Métiers (SAM)}

is an open access repository that collects the work of Arts et Métiers ParisTech researchers and makes it freely available over the web where possible.

This is an author-deposited version published in: https://sam.ensam.eu Handle ID: .http://hdl.handle.net/null

\section{To cite this version :}

Marc BORSENBERGER, Abdelkader BENABOU, Cyrille BAUDOUIN, Régis BIGOT, Pierre FAVEROLLE, Jean-Claude MIPO - Characterization of massive magnetic parts with a dedicated device - International Journal of Applied Electromagnetics and Mechanics - Vol. 57, n4, p.457$472-2018$ 


\title{
Characterization of massive magnetic parts with a dedicated device
}

\author{
Marc Borsenberger ${ }^{\mathrm{a}, \mathrm{b}, *}$, Abdelkader Benabou ${ }^{\mathrm{b}}$, Cyrille Baudouin $^{\mathrm{a}}$, Régis Bigot $^{\mathrm{a}}$, \\ Pierre Faverolle ${ }^{\mathrm{c}}$ and Jean-Claude Mipo ${ }^{\mathrm{c}}$ \\ ${ }^{a}$ LCFC, ENSAM Campus de Metz, 57078 Metz Cedex 3, France \\ ${ }^{\mathrm{b}}$ L2EP, Université des Sciences et des Technologies de Lille, 59655 Villeneuve d'Ascq, France \\ ${ }^{\mathrm{c}}$ Valeo Powertrain Systems, 94046 Créteil Cedex, France
}

\begin{abstract}
Magnetic parts are usually composed of a stack of electrical steel laminations to reduce the eddy current losses. However, for cost reasons or for specific applications the magnetic core can be made from massive steel and thus manufactured with adapted processes such as forging. Such process may lead to inhomogeneous and degraded magnetic properties. Therefore, this study proposes a specific device for characterizing magnetic properties of samples which are to be representative of a massive part.

The measure is based on the Faraday's equation to determine the magnetic flux density and the Hall effect to estimate the magnetic field inside the sample. Practically this is realized with classical components such as Hall probes, a secondary winding and an electromagnet device. However their combination is unique to perform magnetic characterization on massive samples, which are less affected by the sampling technique and may have anisotropic properties.

The device is dimensioned thanks to FE-Simulation and validated according repeatability, sensitivity and trueness analysis. Eventually the characterization is performed on samples with different material parameters showing the effect of the grain size on the specific losses. The expected effect of the grain flow on magnetic properties is however not proven yet.
\end{abstract}

Keywords: FE-Simulation, magnetic characterization, massive sampling, material properties

\section{Introduction}

Usually, in electrical machines, the magnetic core is made from stacked laminations in order to limit the eddy current losses and improve the efficiency of the energy conversion. Nevertheless, for special electrical machines, due to cost reasons in mass production, massive magnetic parts such as the rotor can be incorporated. In that case, the magnetic field is free to circulate in all the bulk, selecting the trajectory that minimizes the energy consumption, or in other words the trajectory with the minimal global reluctance in the electrical machine. This may lead to complex three dimensional trajectories of the flux in the material requiring a specific design of electrical machines with non-trivial geometries of some components of the magnetic circuit. Then, as the performances of electrical machines are strongly related to the magnetic properties of the employed materials, the use of massive magnetic parts requires to pay special attention to the bulk properties of the material [1]. In practice, when designing such electrical

\footnotetext{
${ }^{*}$ Coresponding author: Marc Borsenberger, LCFC, ENSAM Campus de Metz, 4 Rue Augustin Fresnel, 57078 Metz Cedex 3, France. Tel.: +33 3873754 30; Fax: +33 38737 54 70; E-mail: marc.borsenberger@ensam.eu.
} 
devices, numerical tools, such as the finite elements analysis, are usually employed. The material magnetic properties implemented in these tools may not correspond to the real behavior as they are mostly considered as homogeneous and consequently the performances of the simulated electrical device may be different from the experimental one. In fact, the magnetic parts can be issued from complex manufacturing processes that may lead to inhomogeneous properties within the same magnetic part. Therefore, for a more accurate study and/or design of the device, the characterization of the magnetic behavior, by considering the influence of the manufacturing processes, is necessary.

Regarding the massive magnetic parts, they are usually manufactured with a succession of operations including hot forging which impact on the magnetic permeability has been illustrated by Ghodsi and Loghmanian [2]. However, the scientific literature content is mostly dedicated to the study of laminated electrical steels and the related manufacturing processes. For example, the rolling process impact in terms of rolling direction and plastic strain on the magnetic properties has been studied by Landgraf et al. [3] or Hou and Lee [4]. Also, different cutting processes (shearing, laser and wire electric discharge machining) have been compared by Kurosaki et al. [5] in terms of specific losses and magnetizing force to reach $1.5 \mathrm{~T}$. In all these works, phenomenological approaches are adopted and the results may be extended with difficulty to other applications or for numerical modelling.

Another approach consists in considering the inherent material properties, such as the microstructure, mechanical stress or plastic strain, in order to understand the related magnetic properties. In the literature, a correlation between grain size and specific core losses has been revealed in several works [6-8]. Regarding the iron losses, an optimum grain size exists for a given material at a given operating frequency because of the antagonist effects of eddy currents and hysteresis losses. Other studies focus on the effect of mechanical stress and plastic strain on magnetic properties [9-11]. They demonstrate experimentally that slight tensile stresses (for non-pre-stressed laminations) can improve the magnetic properties whereas compression stresses and severe tensile stress deteriorate the magnetic properties. Furthermore, plastic strain, in cold deformation, results in a general flattening of the hysteresis loop and consequently in a deterioration of the magnetic properties.

These observations have largely been made in case of laminated steels because of their large use in magnetic core applications. Moreover, magnetic characterizations are most of the time performed on laminations with techniques such as those described by the IEC 60404 standard [12] (Epstein or Single Sheet Tester). Other non-destructive techniques have also been proposed [13], but remain limited in terms of magnetization level and are not adapted to detail material parameters effect on magnetic properties. For massive magnetic parts, a first approach is the extraction of ring-shaped samples. In that case, the sample may be larger than the zone of interest in the product. Moreover, for this kind of sample, the trajectory of the magnetic flux is circumferential which makes the study of material anisotropies difficult to realize.

In the present work, a dedicated characterization procedure is proposed in order to investigate massive samples properties. The proposed approach consists in extracting, from magnetic steel billets, parallelepipedic samples, which are relatively massive in order to avoid potential mechanical stress relieving and also to limit the impact of edge or surface effects induced by the sampling process. Then, the characterization is performed while the magnetic flux goes though the bulk material and is supposed unidirectional along the sample, allowing the consideration of material anisotropies.

First, the experimental device is described, along with its numerical study to verify the feasibility and the relevance of the measurement setup. Then, the measurement validation is performed on the basis of a sensitivity and repeatability analysis together with a reference measure. Finally, measurements are performed on selected samples, with different mechanical and material anisotropies, to reveal the impact on the magnetic properties. 


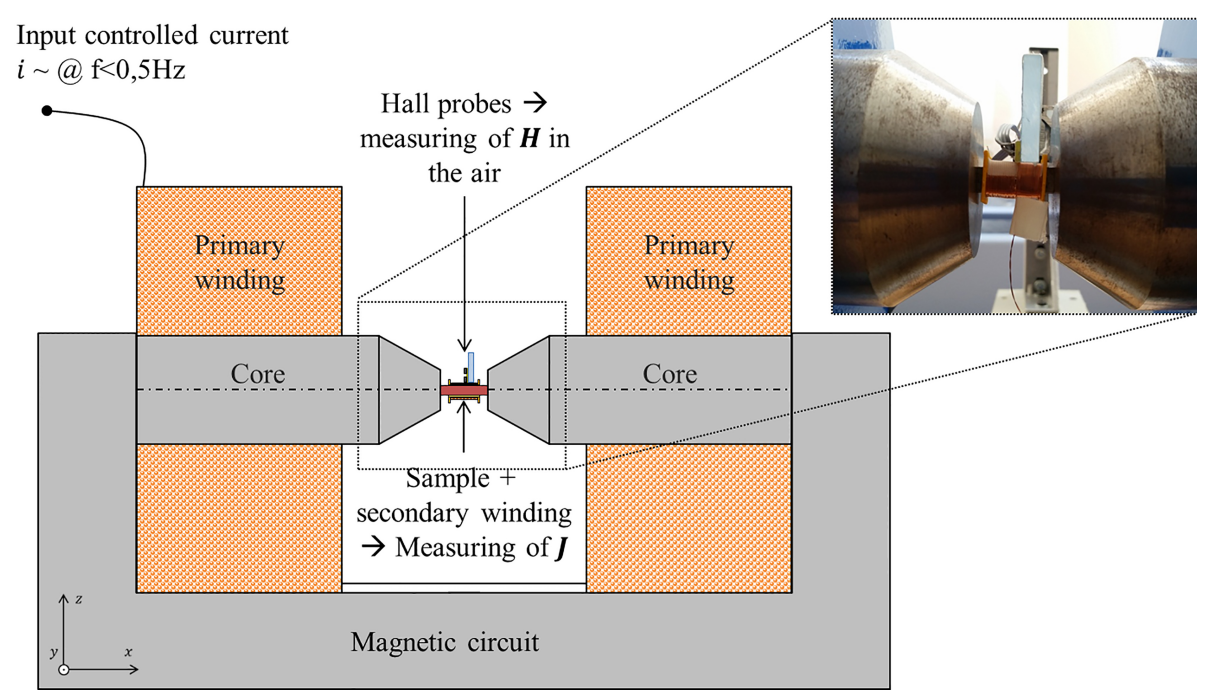

Fig. 1. Schematic of the characterization equipment.

\section{Description of the experimental device}

\subsection{General description}

The magnetic properties of a material describe the evolution of the magnetic polarization $\mathrm{J}$ in function of the excitation magnetic field $\mathrm{H}$ inside the considered sample. In the proposed approach, the sample is placed between the magnetic poles of an electromagnet device (Fig. 1). A controlled current source composed of a function generator (National Instrument USB-6353) and an amplifier (Toellner TOE 7621-60) is connected to the primary winding of the electromagnet device. The amplifier is controlled to generate an alternative current to generate an alternative magnetic field inside the sample.

First, to measure the polarization $\mathbf{J}$ of the sample, a secondary coil is placed on a plastic support in which the sample can be inserted. This way, the eventual dispersion in measurements, due to different windings placed directly on the different samples, is limited. The electromotive force $v_{2}$ is measured with a voltage probe thanks to Lenz-Faraday induction phenomenon. From this measure the magnetic polarization of the sample $J$ is determined with the compensation of the flux in the air:

$$
J=\frac{1}{S_{\text {sample }}}\left(\frac{1}{n_{2}} \int v_{2}(t) d t-\mu_{0} H S_{\text {winding }}\right)
$$

where $S_{\text {sample }}$ is the cross section of the sample, $n_{2}$ and $S_{\text {winding }}$ are respectively the number of turns and the cross-section of the secondary winding, $\mu_{0}$ the magnetic permeability of the vacuum and $H$ the magnetic field estimated in the sample. The magnetic field $H$ is supposed homogeneous in the region enclosed by the secondary winding, that is to say the ferromagnetic sample and the air-gap between the winding and the sample.

To measure the magnetic field $\mathrm{H}$, two Hall probes are placed above the sample. They deliver a voltage proportional to the magnetic field $H$ at two known locations in the air around the sample. As a first approach, a linear extrapolation is chosen to estimate the magnetic field at the surface of the sample:

$$
H=\frac{H_{\text {probe } 1} z_{\text {probe 2 }}-H_{\text {probe } 2} z_{\text {probe } 1}}{z_{\text {probe 2 }}-z_{\text {probe } 1}}
$$




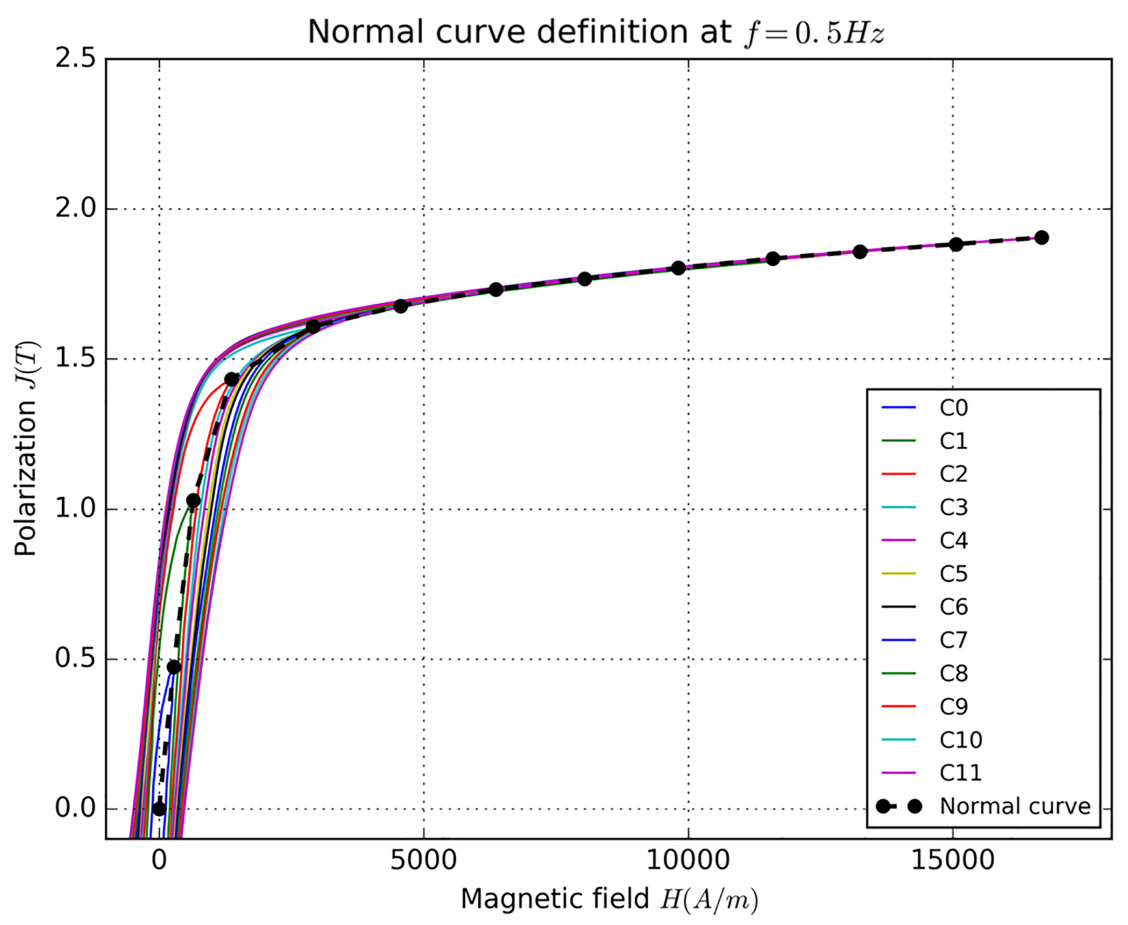

Fig. 2. Normal curve extraction from centered loops.

where $H_{\text {probe i }}$ and $z_{\text {probe i }}$ are respectively the magnetic field measured by the probe $i$ and its distance to the sample surface. The direct output of such a fluxmetric technique is an hysteresis loop whereas generally, the electromagnetic numerical tools require the use of a single-valued behavior law of the materials. Therefore, from the measured hysteresis loops, the normal polarization curve will be extracted. This curve is drawn from the maximum magnetic fields and polarizations of centered inner loops measured at different levels of polarization, as illustrated in Fig. 2.

In addition, the specific core losses are determined in our case for a given applied magnetic field $H$ :

$$
\mathcal{P}_{H[k A / m]}[W / k g]=\frac{f}{\rho} \int H d B
$$

where $f$ is the frequency in $\mathrm{Hz}, \rho$ the material density in $\mathrm{kg} / \mathrm{m}^{3}$ and $B$ the magnetic flux density in $T$.

Several considerations are observed regarding the experimental device. First of all, the standards recommend to measure a sinusoidal magnetic flux density whereas in the proposed technique the input current is sinusoidal. In fact, in the quasi-static conditions, the hysteresis behavior is more dependent on the extreme values of the magnetic field than on its waveform. Moreover, the sample is $25 \mathrm{~mm}$ length and has a square basis of $8 \mathrm{~mm}$ wide, which is considered massive regarding the dimensions of steel sheets used in Epstein frames. In addition, the extreme surfaces of the parallelepipedic sample must be as parallel and as flat as possible to limit the parasite air gaps which would increase the leakage flux around the sample and introduce a strong gradient of the magnetic field $H$ between the poles of the electromagnet device. Eventually the frequency must remain low $(<0.5 \mathrm{~Hz})$ because of the design of the electromagnet and also to avoid skin effects in the sample. Since the material investigated is a soft magnetic steel grade, these considerations lead indeed to a skin depth of about $10 \mathrm{~mm}$ which is sufficient, regarding half of the width of the sample, to neglect the skin effect. 


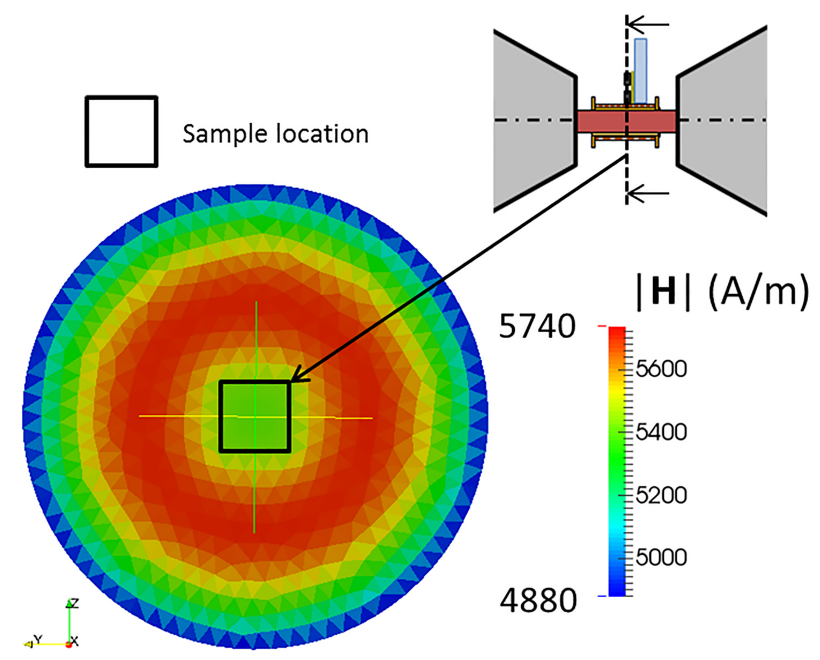

Fig. 3. Distribution of the magnetic field $\mathrm{H}$ between the poles of the electromagnet according to the FE simulation.

\subsection{Numerical study of the device}

A first study of the experimental device is performed through a numerical modelling approach. The three dimensional electromagnetic problem is solved thanks to a time stepping finite element (FE) method and using electric potential formulation to consider the electrical conductivity in the sample. The magnetic behavior law of the sample used in the model is similar to what is expected for the considered material.

From this model, an investigation on several hypotheses can be performed in order to improve the understanding of the different sources of errors. The first hypothesis considers that the magnetic field $H$ and the magnetic flux $\Phi$ are measured at the same position. In fact, the Hall probes measure the magnetic field at two points in the space surrounding the sample and along a given direction, whereas the secondary winding gives the average magnetic flux density which passes through the surface of the winding and on a given length of the winding. The Eq. (1) also supposes that the magnetic field in the sample and in the air gap between the winding and the sample is the same.

The numerical simulation shows that the magnetic field surrounding the sample is not homogeneous. In Fig. 3, the distribution of the magnetic field is shown in a cross-section located in the middle of the sample between the electromagnet poles.

The homogeneous center of this distribution $(\approx 5300 \mathrm{~A} / \mathrm{m})$ represents the sample. Then, by increasing the distance to the sample, the magnetic field increases to a maximum and decreases. This calculated magnetic field profile is verified experimentally by moving the Hall probes along the Z-axis (back to Fig. 1).

The scatter plot of Fig. 4 (blue diamonds) represents, for a given level of the excitation field, the measured profile of the magnetic field $H$ above the sample, from the upper surface of the sample (origin, vertical green line) to the limit of the flat surfaces of the pole (vertical dashed line). In this particular configuration, the magnetic field $\mathrm{H}$ inside the sample is estimated with more accuracy thanks to a polynomial fitting. The corresponding reference value of the magnetic field $H$ inside the sample is the evaluation of the polynomial function at the origin.

During the magnetic characterization, the linear extrapolation from Eq. (2) is made from the two positions of the Hall probes represented by the two red points in Fig. 4. The relative error with the reference value is estimated at $1.32 \%$. 
Hall probes position effect on magnetic field measurement

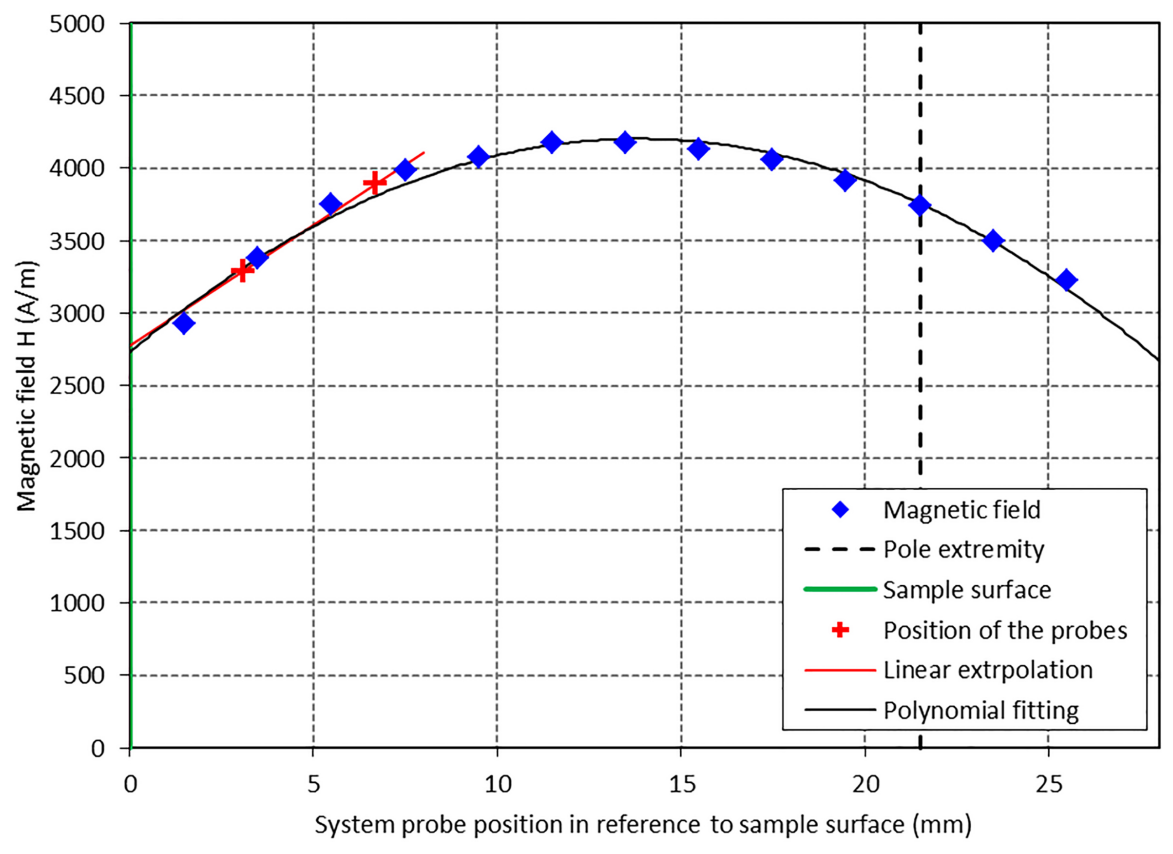

Fig. 4. Magnetic field distribution above the sample : experimental measures, polynomial fitting for the reference value and current linear extrapolation method during characterization.

However, the air gap between the electromagnet poles and the sample is inevitable. A simulation has been performed to verify the impact of the air gap on the magnetic field $H$ and its estimation.

Figure 5 shows the simulated profile of the magnetic field above the sample for an air gap of $0.15 \mathrm{~mm}$ between each electromagnet pole and the two extreme surfaces of the sample. The magnetic field $H$ is estimated either with linear extrapolation according to Eq. (2) or with a third-order polynomial Lagrangian extrapolation and using four virtual measures of the magnetic field. In that specific case, the error on the estimation of the magnetic field can be reduced from $425 \%$ to $105 \%$.

Eventually, another assumption is a uniform distribution of the magnetic flux density in the sample in every cross section along the length of the secondary winding which is confirmed by the simulation.

\section{Uncertainty analysis of the measure}

\subsection{Sensitivity and repeatability}

To evaluate the uncertainty of the measure, two steps are performed: a qualitative analysis and a comparison with the classical ring core characterization technique.

The qualitative analysis is represented by the ability of the characterization protocol to give always the same result (precision according Standard ISO 5725-1). The major source of errors is related to the relative positions of the sample, the electromagnet device, the probes and the secondary winding. A preliminary experimental sensitivity analysis has been performed. The effect of the following parameters has been investigated:

- The Hall probes position regarding the sample: along X, Y and Z-axis (Fig. 1), 


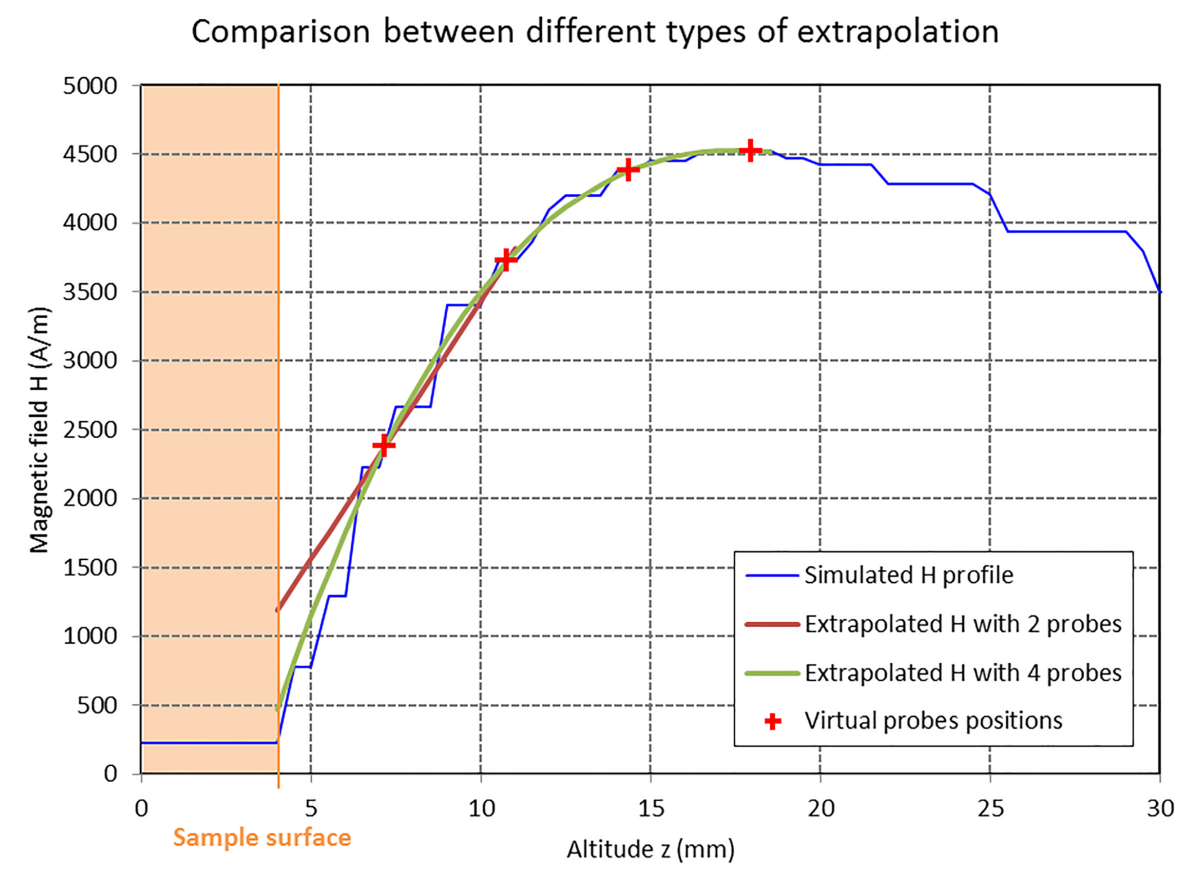

Fig. 5. Estimation of magnetic field with different extrapolations (Simulation results, air gap $=2 \times 0.15 \mathrm{~mm}$ ).

- The secondary winding position: centered or in contact with a pole (along $\mathrm{X}$ direction),

- The sample position in the magnetic poles

It has been observed that the secondary winding position has a minor impact on the measures whereas the relative position of the Hall probes, sample and electromagnet poles must be accurate. Indeed the Hall probes must be as close to the sample as possible in order to limit the error due to the linear extrapolation to estimate the magnetic field at the sample surface.

To go further in the qualitative analysis, a set of ten measurements are performed on the same sample under repeatability conditions. The following protocol is applied for each measurement:

- Positioning the sample between the magnetic poles,

- Compensating the remnant induction in the magnetic circuit for a given amplitude of the excitation field,

- Recording the monitored quantities for ten different levels of the input current,

- Post-processing of the measures.

The results associated to the normal curves are shown in Fig. 6 and those associated to the losses, at an excitation field of $16 \mathrm{kA} / \mathrm{m}$, in Fig. 7.

Qualitatively the Fig. 6 shows slight variations on the normal curves. Considering the $\pm 2 \sigma$ interval, where $\sigma$ is the standard deviation, the variations in the result is mostly due to the uncertainty on the measure of the magnetic field $\mathrm{H}$. For example, to compare numerically the normal curves, and as a first approach, the chosen criterion is the required magnetic field $H_{1.5}$ to reach a magnetic polarization of $1.5 \mathrm{~T}$. Considering a cubic interpolation between the measured points, the repeatability of the measurement can be qualified with an average value of $\bar{H}_{1.5}=2370 \mathrm{~A} / \mathrm{m}$ and a standard deviation of $\sigma=$ $99 \mathrm{~A} / \mathrm{m}$, representing a coefficient of variation $\sigma / \bar{H}=4.2 \%$. Concerning the specific losses at $16 \mathrm{kA} / \mathrm{m}$ shown in Fig. 7, the coefficient of variation $\sigma / \bar{\rho}$, with $\bar{\rho}$ the average loss value, represents $2.13 \%$. 


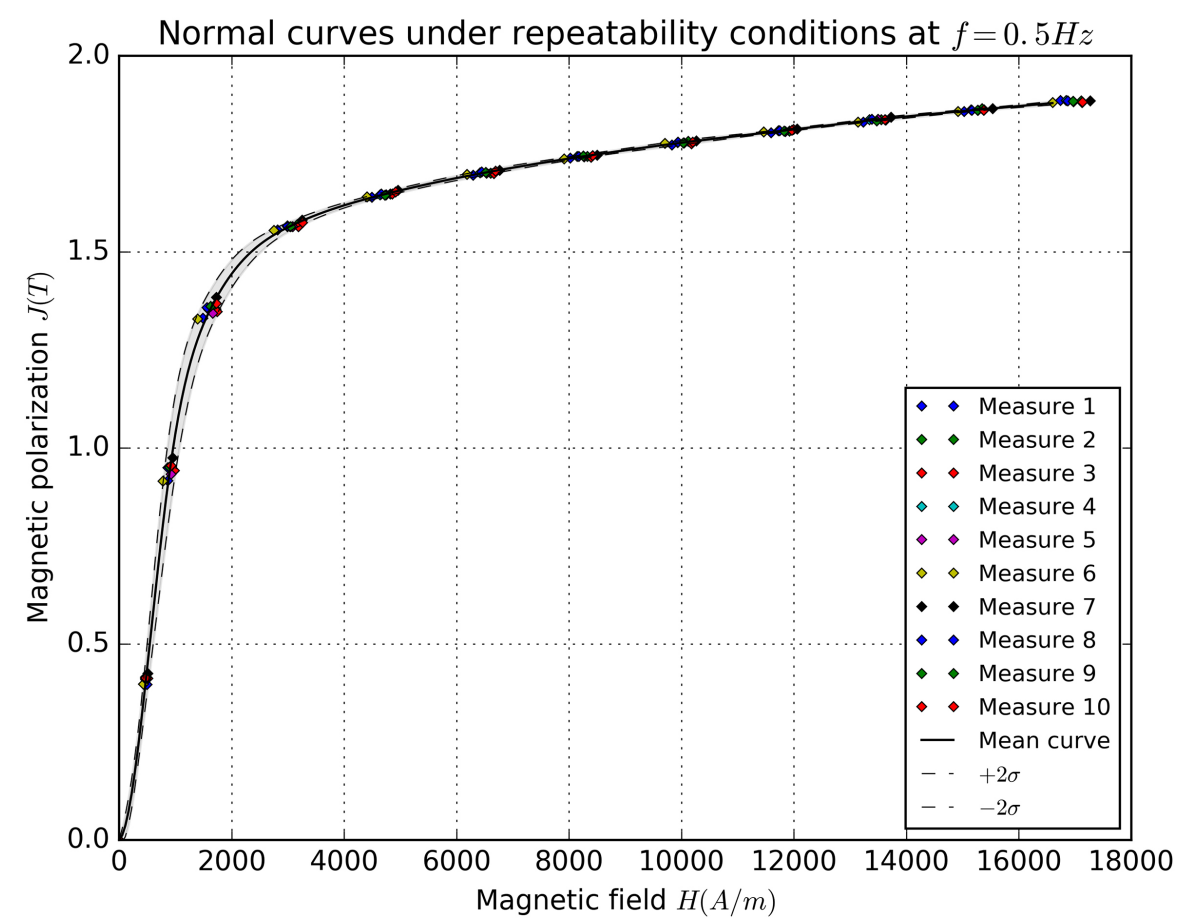

Fig. 6. Normal curves under repeatability conditions for the considered test sample.

Note that, to distinguish the effect of material parameters on the magnetic properties, the calculated error of precision must be significantly inferior to the variations observed on samples with different magnetic properties.

\subsection{Comparison with another characterization methodology}

The precision of the measure has been investigated in the previous section. The next parameter to be considered to estimate the uncertainty of the measure with the characterization device is the trueness (according Standard ISO 5725-1). This parameter describes the deviation between the measured and the true value.

To estimate the trueness of the measure, the results of magnetic characterization with the presented device are compared to the standard ring core measurement. Measures with both techniques have been performed on reference samples, taken from the same hot rolled bar in two consecutive slices as presented on the diagram from Fig. 8.

The material anisotropies and eventual variations in the material properties, due to hot rolling and impact of the sampling technique, must be taken into account. In this kind of billet, mechanical anisotropy is often qualified with the grain flow, that is to say the elongation of the malleable inclusions in the matter in the same direction as the flow of matter during the forming process [14]. Hence the reference samples are taken in a way, described in Fig. 8, that the magnetic flux has a similar trajectory orientation regarding the grain flow in the billet. Also, the locations of the samples and the ring core are chosen at a similar radius in order to limit the impact of an eventual variation of the material properties (such as the microstructure) along the radius. These samples are furthermore obtained using wire electric discharge machining, which is assumed to have a repeatable and limited impact on the magnetic properties [5]. 


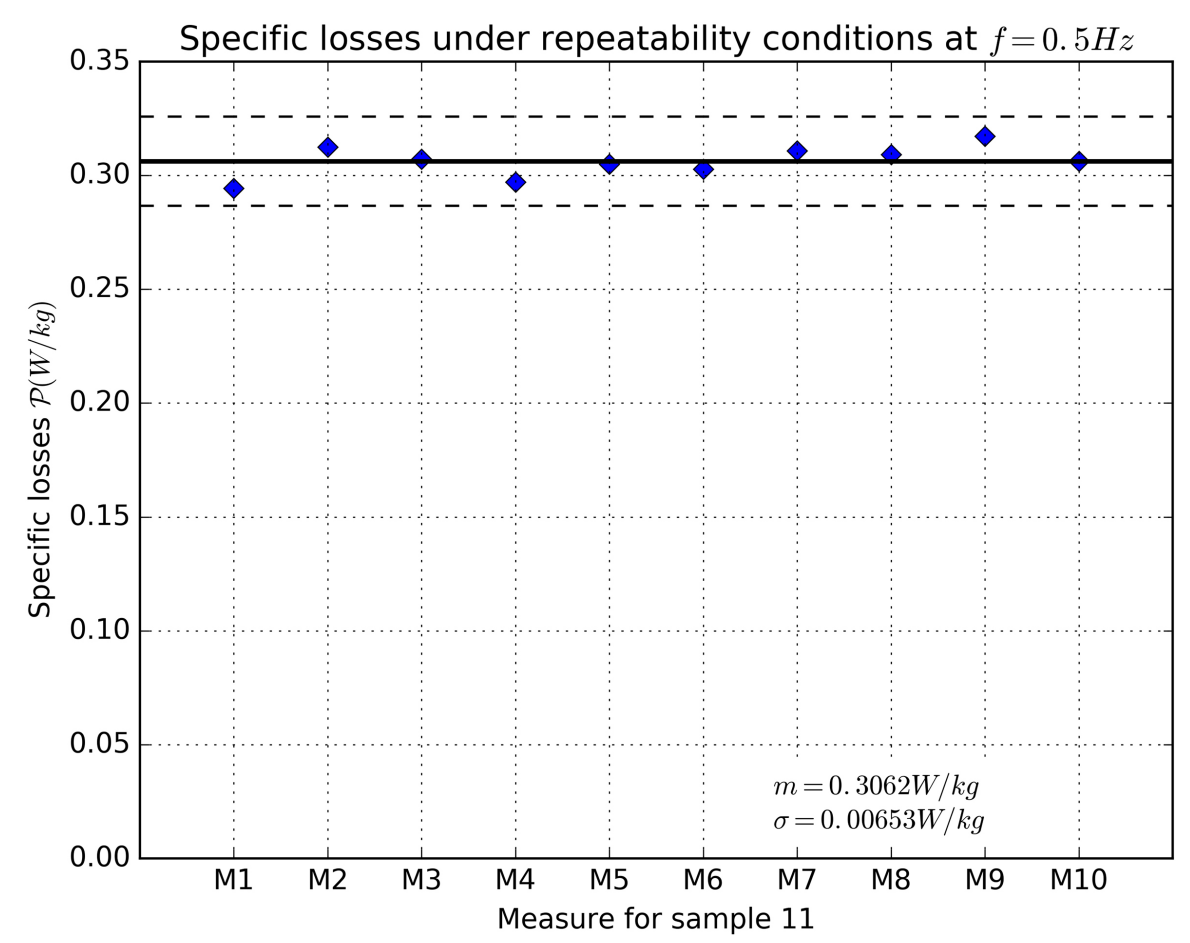

Fig. 7. Specific losses under repeatability conditions for the considered test sample.

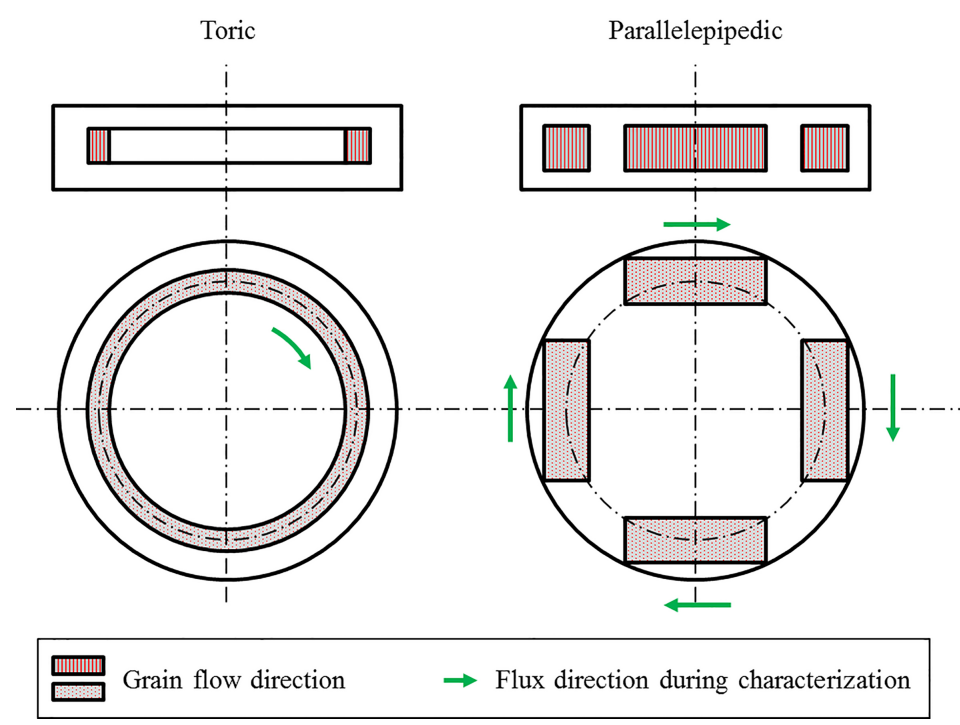

Fig. 8. Position of the reference samples in two consecutive slices, flux direction during characterization and grain flow direction.

The experimental protocol consists in repeating three times the measurements for each parallelepipedic sample at twelve levels of the input current. The normal curves obtained for the samples are shown in Fig. 9 where $R_{i}$ stands for the sample number and $M_{i}$ for the measure repetition. 


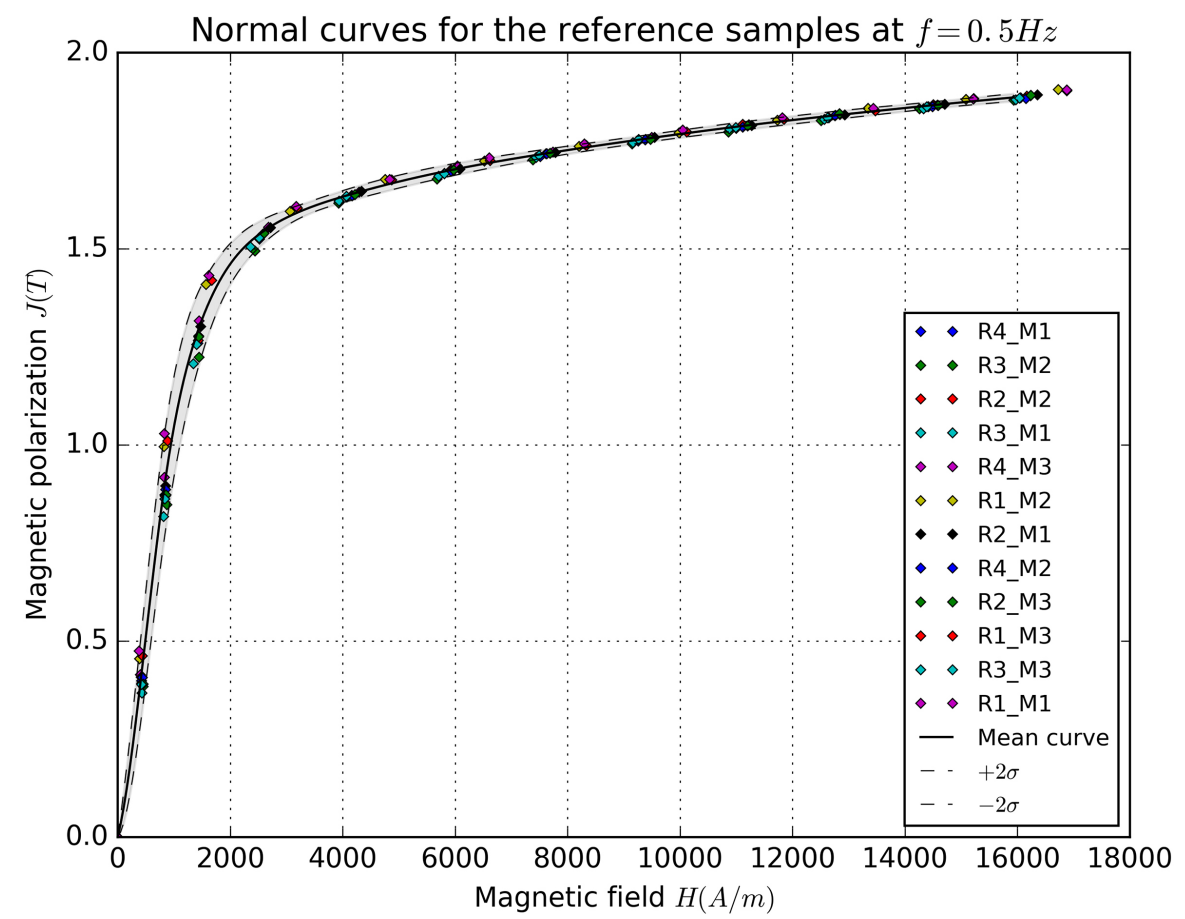

Fig. 9. Normal curves for the parallelepipedic reference samples.

The magnetic field needed to reach a polarization level of $1.5 \mathrm{~T}$ has an average value of $2190 \mathrm{~A} / \mathrm{m}$ and a standard deviation of $142 \mathrm{~A} / \mathrm{m}$ over these twelve measurements, that is to say a coefficient of variation of $6.7 \%$. The average value of the specific losses is $0.248 \mathrm{~W} / \mathrm{kg}$ and the associated standard deviation is $0.011 \mathrm{~W} / \mathrm{kg}$ over the twelve measures, representing a coefficient of variation of $4.4 \%$. This represents slightly more variation than the repeatability measures. The conclusion is that the parallelepipedic reference samples are slightly different from each other in terms of specific losses and magnetic field $H_{1.5}$.

Regarding the ring core samples, the measure has been performed to investigate about the relevancy and limits of the dedicated characterization device. Three ring cores of dimensions D50 $\mathrm{mm} \times \mathrm{d} 42 \mathrm{~mm} \times$ $\mathrm{h} 6 \mathrm{~mm}$ have been extracted from the same bar as the parallelepipedic samples (Fig. 8). The primary and the secondary windings have been designed to reach a comparable magnetic field range. Each ring core is measured three times. The characterization has been made using a Brockhaus MPG200 measurement equipment which controls the magnetic flux density B in closed-loop. Nine levels of amplitudes are reached from $0.2 \mathrm{~T}$ to $1.8 \mathrm{~T}$ at $3 \mathrm{~Hz}$. In that case the skin depth is about $4 \mathrm{~mm}$ which is still acceptable to neglect this effect regarding the sample dimension. From the measured hysteresis loops, the bench gives the information on the normal curve directly. In this kind a measurements, the magnetic quantities are usually evaluated according to the following formulas:

$$
\begin{aligned}
H & =\frac{n_{1} i}{l_{m}} \\
J & =\frac{1}{n_{2} S_{\text {Sample }}} \int v_{2}(t) d t-\mu_{0} H
\end{aligned}
$$

where $n_{1}$ and $n_{2}$ are the number of turns of respectively the primary and secondary winding, $l_{m}$ the 
Normal curves for parallelepipedic and ring core samples

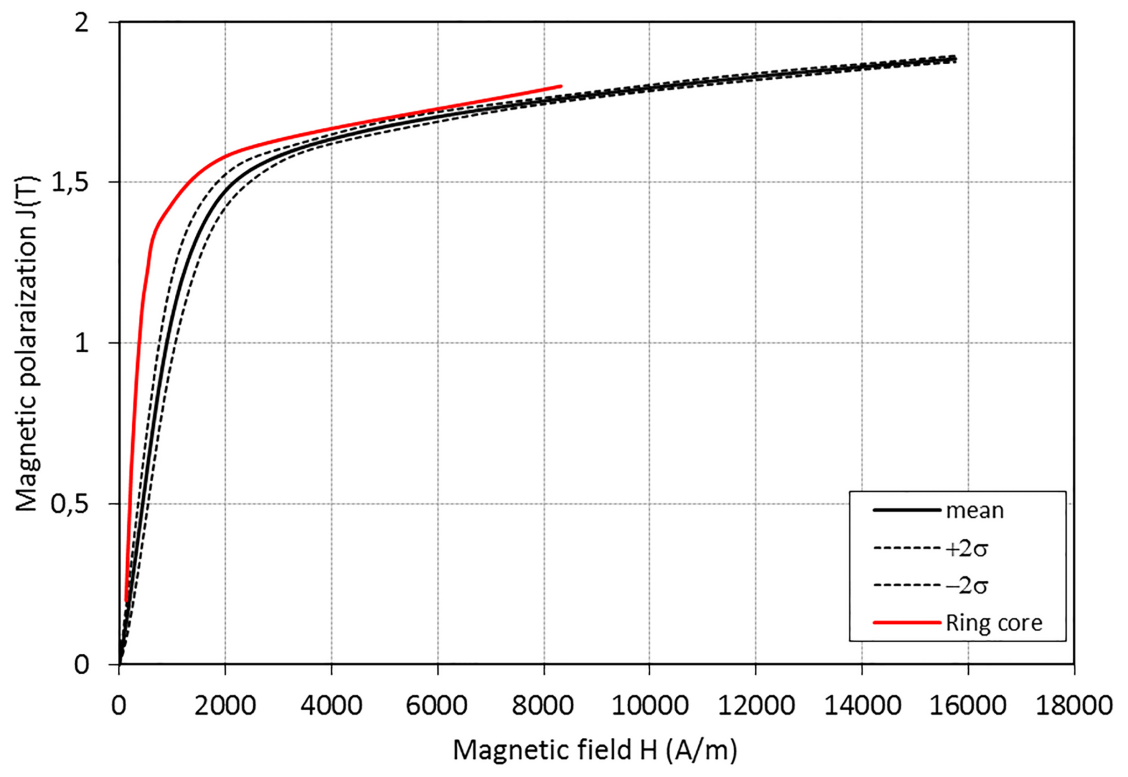

Fig. 10. Comparison between the reference samples and the ring core.

characteristic length of a magnetic core according to Standard IEC 60205. Here the secondary winding cross section is assumed to be the same as the sample cross section.

The comparison between the normal curves from the used dedicated device and standard ring core characterizations are shown in Fig. 10.

In the standard measures (ring core), the required field to reach $1.5 \mathrm{~T}$ is $H_{1.5}=1425 \mathrm{~A} / \mathrm{m}$. In that case, the specific characterization gives a value about $50 \%$ superior. This difference can be explained by different sources of deviations. Firstly, the samples are not exactly the same. Despite the sampling methodology presented before, they have not been taken exactly at the same place, so that their material properties, in terms of grain size, plastic strain or grain flow may differ from one sample to another. Moreover after the sampling process the residual stresses can be relaxed in a different way due to the closed shape of the ring core and the open shape of the parallelepipedic sample.

Secondly, the main source of deviation is due to the characterization procedure. On the one hand, at high level of magnetic field i.e. in the saturation zone, the standard ring core measure becomes inaccurate, for instance because of the inter-turns leakage flux and the axial component of the magnetic field. Furthermore, for these measures the compensation of the flux in the air gap between the sample and the secondary winding is not taken into account, and the compensation of the axial magnetic field is not guaranteed. In the other hand, the error can be imputed to several hypotheses enounced in the case of the specific characterization. First one major source of deviation is the linear extrapolation which overestimates the magnetic field $\mathrm{H}$ at the sample surface inside the sample whereas the profile shape is parabolic. The difference increases with a more significant gradient of the magnetic field $\mathrm{H}$ between the electromagnet poles. This is the case at low levels of magnetic field $\mathrm{H}(<1000 \mathrm{~A} / \mathrm{m})$ or if the magnetic flux is not well channeled through the sample, for example if the air gap between the sample and the poles increases or if the magnetic properties are not sufficiently high. Secondly, the magnetic flux is averaged on the total length of the secondary winding. This leads to an uncertainty on the estimation of the magnetic polarization $\mathrm{J}$ which can shift the normal curve from the reference normal curve. 
Table 1

Magnetic field $H_{1.5}$ for different grain sizes

\begin{tabular}{lccc}
\hline Grain size & $9(15 \mu \mathrm{m})$ & $6(45 \mu \mathrm{m})$ & $0(350 \mu \mathrm{m})$ \\
\hline$\overline{H_{1.5}}$ & $2350 \mathrm{~A} / \mathrm{m}$ & $2250 \mathrm{~A} / \mathrm{m}$ & $2200 \mathrm{~A} / \mathrm{m}$ \\
$\sigma\left(H_{1.5}\right)$ & $200 \mathrm{~A} / \mathrm{m}$ & $160 \mathrm{~A} / \mathrm{m}$ & $220 \mathrm{~A} / \mathrm{m}$ \\
\hline
\end{tabular}

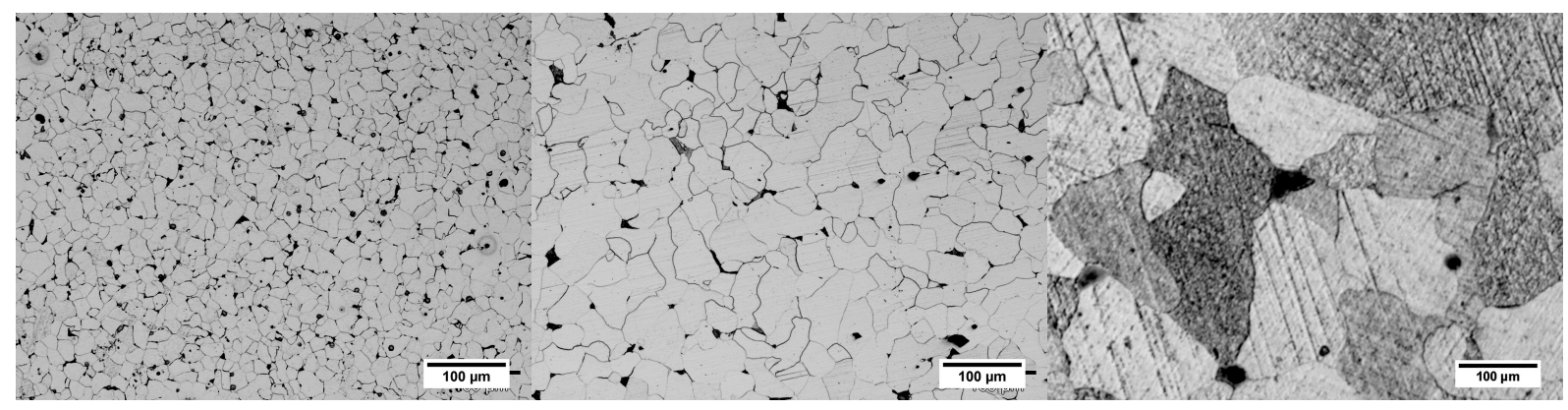

Fig. 11. Micrographs for the two different sets of samples (left: set A; middle: set B; right: set C).

In conclusion, a deviation between the ring core measurement and the presented technique has been observed. However comparative studies can be performed with this device considered the evaluated repeatability error. Consequently characterizing different samples using this technique reveals the impact of material parameters on magnetic properties.

\section{Application to massive magnetic samples having different material properties}

As explained in the introduction, the manufacturing process, like hot forging, has a strong impact on material and mechanical properties. The proposed characterization approach is now used to study the impact of these inherent properties on magnetic properties. The sensitivity of the device is illustrated with selected material parameters. On one hand, the grain size influence on magnetic properties, especially the specific losses, is verified and on the other hand, the influence of grain flow on the magnetic properties is investigated.

\subsection{Grain size}

Three sets of samples are investigated in this section. The first one (set A) is directly sampled in a hot rolled cylindrical bar, the corresponding grain size index is 6 ASTM $(45 \mu \mathrm{m})$. The two other are obtained after different heat treatments. The set B has been obtained with a normalizing heat treatment, at a temperature of $900^{\circ} \mathrm{C}$ during 10 minutes with air cooling. The obtained grain size index is 9 ASTM $(15 \mu \mathrm{m})$. The set $\mathrm{C}$ has been obtained with a grain growth heat treatment, at a temperature of $1100^{\circ} \mathrm{C}$ during 20 hours and furnace cooling. The obtained grain sized index is about 0 ASTM $(350 \mu \mathrm{m})$. The grain size is evaluated using the intercept segment method according the standard ISO 643. The corresponding micrographs are shown in Fig. 11.

The samples have been magnetically characterized with the proposed technique. The results concerning the magnetic field needed to reach a polarization of $1.5 \mathrm{~T}$ are shown in Table 1.

Considering only the mean values, these results may show a trend that the larger the grain is, the lower the magnetic field $H_{1.5}$ is, which describes better magnetic properties. However, the standard deviation of these measures prevents an evident conclusion. 
Table 2

Specific losses at $0.5 \mathrm{~Hz}$ and $16 \mathrm{kA} / \mathrm{m}$ for different directions

\begin{tabular}{ccc}
\hline$\theta$ & $0^{\circ}$ & $90^{\circ}$ \\
\hline$\overline{\mathcal{P}_{16}}$ & $0.240 \mathrm{~W} / \mathrm{kg}$ & $0.247 \mathrm{~W} / \mathrm{kg}$ \\
$\sigma\left(\mathcal{P}_{16}\right)$ & $0.015 \mathrm{~W} / \mathrm{kg}$ & $0.011 \mathrm{~W} / \mathrm{kg}$ \\
\hline
\end{tabular}

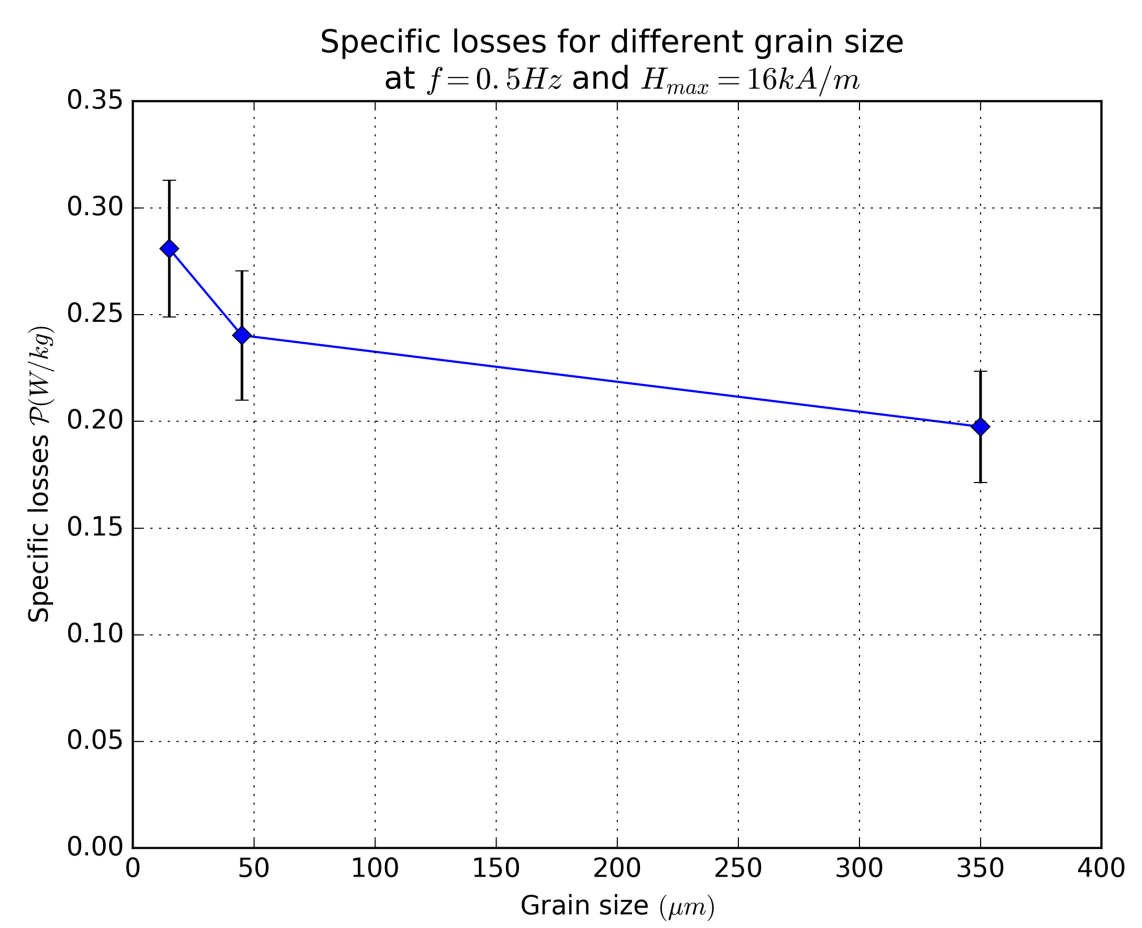

Fig. 12. Influence of the grain size on specific losses.

Regarding the specific losses, the results in Fig. 12 show a clear trend that these losses decrease with larger grains. Considering the given frequency of $0.5 \mathrm{~Hz}$, the eddy current losses seems not significant and a diminution of the hysteresis losses with larger grain size is expected as found in the literature [6].

\subsection{Grain flow}

As discussed in the introduction, the literature shows difference in terms of magnetic properties according to the direction of characterization regarding the rolling direction for electrical steel laminations. The phenomenon is significant between the two cross direction $0^{\circ}$ and $90^{\circ}$ [3]. In this case, due to the hot rolling process, the fiber is oriented in the same direction as the cylindrical bar axis. To evaluate the impact of the grain flow, two sets of samples are taken from the billet according different directions represented in Fig. 13.

These samples have been characterized with the presented device at $0.5 \mathrm{~Hz}$ and the same 12 levels of input current. The Table 2 shows the specific losses at $16 \mathrm{kA} / \mathrm{m}$ for the two sets of investigated samples. The distance between the two sets is not significant regarding the associated standard deviations.

The Fig. 14 shows the mean normal curves for the two sets of samples. For the direction $\theta=0^{\circ}$, the magnetic field required to reach $1.5 \mathrm{~T}$ of polarization is $H_{1.5}=2250 \mathrm{~A} / \mathrm{m}$ with a standard deviation of $160 \mathrm{~A} / \mathrm{m}$. Concerning the direction $\theta=90^{\circ}$, the respective values are $2190 \mathrm{~A} / \mathrm{m}$ and $142 \mathrm{~A} / \mathrm{m}$. 


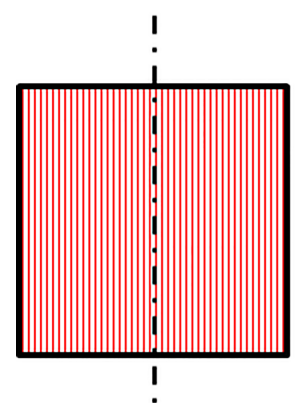

Initial billet

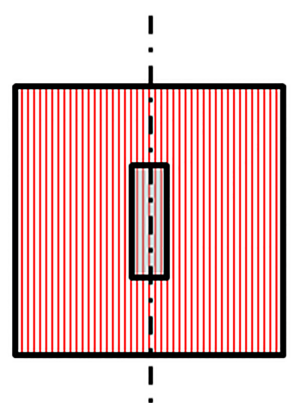

$\theta=0^{\circ}$

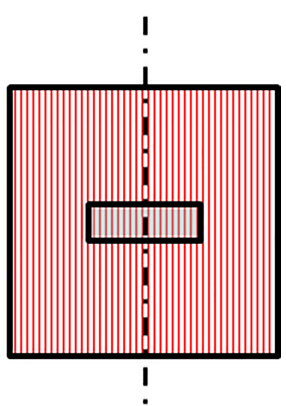

$\theta=90^{\circ}$

Fig. 13. Sampling with different angles to grain flow direction.

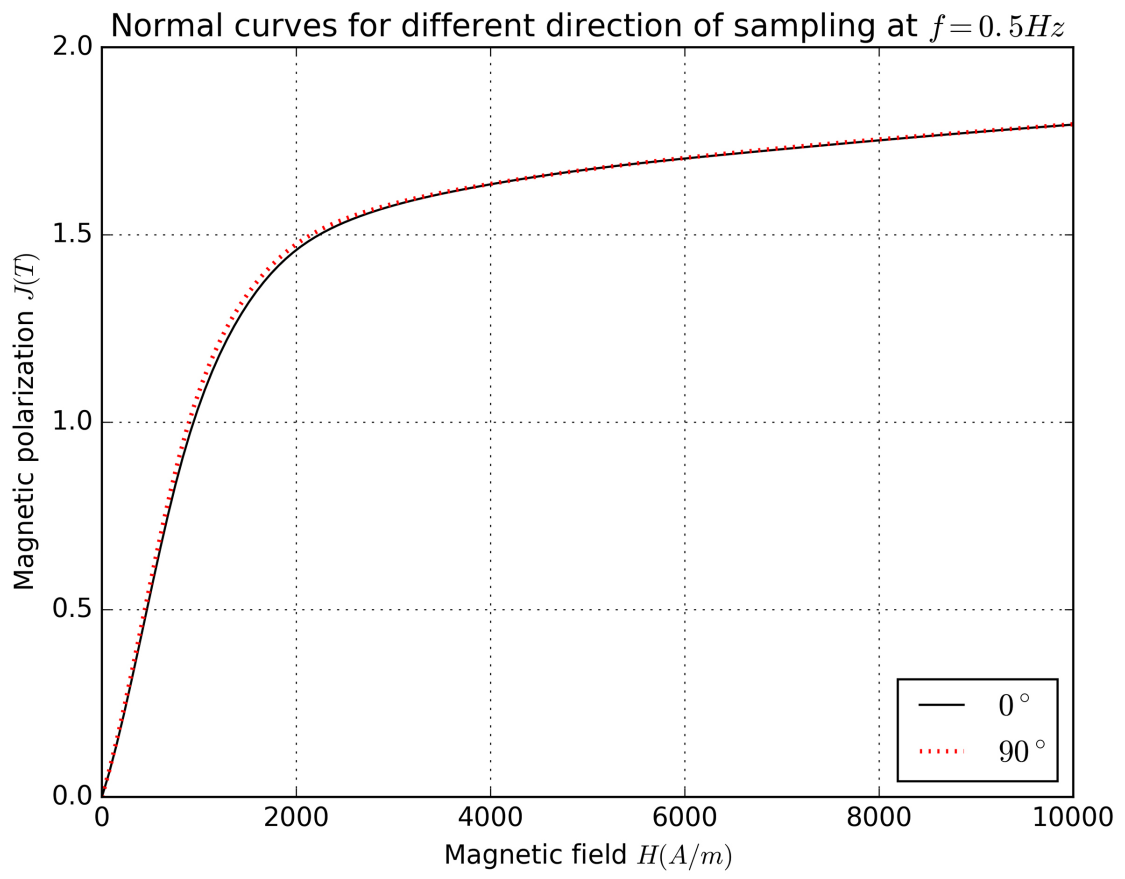

Fig. 14. Normal curve for different direction of sampling.

The proposed specific protocol allows to verify that, for this steel grade (with a very low content of element of addition) for the frequency of $0.5 \mathrm{~Hz}$, grain flow has no significant impact on magnetic properties.

\section{Conclusion}

The present work introduces the investigation of a dedicated magnetic characterization device to assess the influence of material properties. The literature shows many results in terms of impact of the material properties and processes on magnetic properties for laminated samples. The objective of the proposed 
approach is to extend all these results to massive samples, and keeping the possibility to investigate material anisotropies.

The presented device rests on a classic method of magnetic flux measurement and magnetic field estimation. The Section 3.1 confirms that the device is able to give repeatable measures. Nevertheless, the difference with the measure on ring cores revealed that the magnetic field $\mathrm{H}$ seems to be overestimated and the measures are not quantitative yet. Several trays of improvement of the characterization technique can be tested. The addition of more Hall probes to estimate the quadratic profile of the magnetic field within the electromagnet poles can reduce the error due to the linear extrapolation. Modifying the magnetic circuit topology or adding a magnetic shielding can also positively impact the spatial evolution of the magnetic field and make the linear extrapolation relevant.

At the end of this work, an application of the device has been proposed in order to study the impact of material properties on magnetic properties. Among the different sets of samples investigated, the standard deviation of the magnetic field $H_{1.5}$ remains slightly greater than the error of repeatability. The samples inside a same set have consequently similar magnetic respectively material properties. For the studied material, the influence of the material properties on the normal curves and the magnetic field $H_{1.5}$ are not significant for the investigated parameters. However, it has been shown that the grain size has a significant impact on specific losses, which is in accordance with the literature.

The illustration can be extended to other material parameters which are also investigated in the literature, such as crystallographic texture, plastic strain or residual stress. Investigating other steel grades with more element of addition result in more pronounced inclusions and may show differences of magnetic properties according to grain flow.

The immediate perspective of the work consists in improving the protocol with the proposed solutions and to apply the methodology to other material parameters in order to establish empiric models to predict their influence on magnetic properties.

\section{References}

[1] L. Li, A. Kedous-Lebouc, A. Foggia and J.-C. Mipo, Influence of Magnetic Materials on Claw Pole Machines Behavior, IEEE Trans Magn 46(2) (Feb. 2010), 574-577.

[2] M. Ghodsi and S.M.R. Loghmanian, Effect of forging on ferromagnetic properties of low-carbon steel, 2011, pp. 1-3.

[3] F.J.G. Landgraf, M. Emura, J.C. Teixeira and M.F. de Campos, Effect of grain size, deformation, aging and anisotropy on hysteresis loss of electrical steels, J Magn Magn Mater 215-216 (Jun. 2000), 97-99.

[4] C.-K. Hou and S. Lee, Effect of rolling strain on the loss separation and permeability of lamination steels, IEEE Trans Magn 30(2) (Mar. 1994), 212-216.

[5] Y. Kurosaki, H. Mogi, H. Fujii, T. Kubota and M. Shiozaki, Importance of punching and workability in non-oriented electrical steel sheets, J Magn Magn Mater 320(20) (Oct. 2008) 2474-2480.

[6] M.F. de Campos, J.C. Teixeira and F.J.G. Landgraf, The optimum grain size for minimizing energy losses in iron, J Magn Magn Mater 301(1) (Jun. 2006), 94-99.

[7] R. PremKumar, I. Samajdar, N.N. Viswanathan, V. Singal and V. Seshadri, Relative effect(s) of texture and grain size on magnetic properties in a low silicon non-grain oriented electrical steel, J Magn Magn Mater 264(1) (Aug. 2003), 75-85.

[8] E.J. Gutiérrez-Castañeda and A. Salinas-Rodríguez, Effect of annealing prior to cold rolling on magnetic and mechanical properties of low carbon non-oriented electrical steels, J Magn Magn Mater 323(20) (Oct. 2011), 2524-2530.

[9] V.E. Iordache, E. Hug and N. Buiron, Magnetic behaviour versus tensile deformation mechanisms in a non-oriented Fe-(3 wt.\%)Si steel, Mater Sci Eng A 359(1-2) (Oct. 2003), 62-74.

[10] L. Bernard and L. Daniel, Effect of Stress on Magnetic Hysteresis Losses in a Switched Reluctance Motor: Application to Stator and Rotor Shrink Fitting, IEEE Trans Magn 51(9) (Sep. 2015), 1-13.

[11] L. Vandenbossche, S. Jacobs, D. Van Hoecke and E. Attrazic, Impact of mechanical stresses on the magnetic performance of non-oriented electrical steels and its relation to electric machine efficiency, in: 2015 IEEE Transportation Electrification Conference and Expo (ITEC), 2015, pp. 1-6. 
[12] IEC, IEC 60404-2:1996 - Magnetic materials - Part 2: Methods of measurement of the magnetic properties of electrical steel sheet and strip by means of an Epstein frame. 1996.

[13] L. Arbenz, A. Benabou, S. Clénet, J.-C. Mipo and P. Faverolle, Characterization of the local incremental permeability of a ferromagnetic plate based on a four needles technique, in: SMM, 2015.

[14] ASM International, ASM Handbook: Volume 11, Failure analysis and prevention. Ohio: ASM International, 1986. 